\title{
КОРРЕКЦИЯ КЛИНИЧЕСКИХ ПРОЯВЛЕНИЙ ЭНДОМЕТРИОЗА И НАРУШЕНИЙ МЕНСТРУАЛЬНОГО ЦИКЛА
}

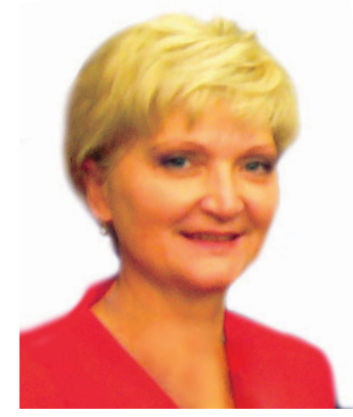

Г.Ф. РОЩИНА

к.мед.н., доцент кафедры акушерства и гинекологии № 1 Национальной медицинской академии последипломного образования им. П.Л. Шупика $\ni^{\prime}$ ндометриоз - единственный в своем роде дисгормональный процесс, обладающий способностью к инфильтративному росту и метастазированию. Это заболевание характеризуется разрастанием эндометриоидной ткани за пределами эндометрия, которая по морфологическим признакам подобна слизистой оболочке матки [1-3, 7].

Термин «эндометриоз» впервые предложил Blair Bell в 1892 г. Эндометриоидную ткань нельзя рассматривать как эктопически расположенный эндометрий, так как они существенно различаются по содержанию рецепторов к гормонам, ответу на гормональное воздействие и по визуальным проявлениям [1, 3, 6].

Актуальность проблемы эндометриоза обусловлена возрастающей его распространенностью, повышением частоты бесплодия при его наличии, формированием стойкого болевого синдрома при некоторых фрормах, а также увеличением числа наблюдений «острого живота». Эндометриоз чаще всего встречается у женщин репродуктивного возраста 25-40 лет. Эндометриоидные кисты (эндометриомы) в среднем образуются у пациенток до 30 лет, внутренний эндометриоз - в 40 лет [5, 16, 17, 29, 30].

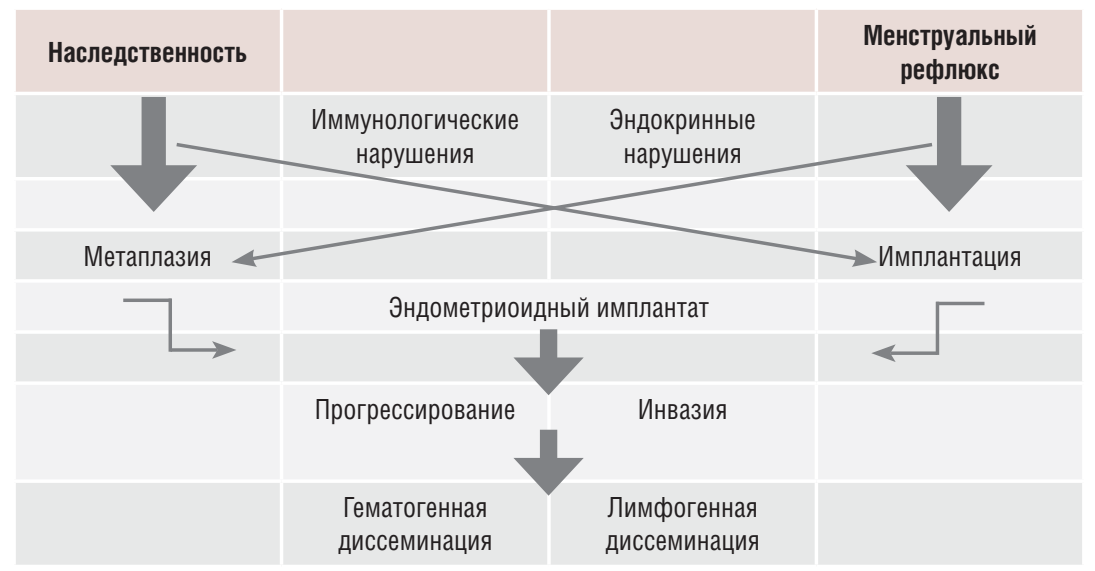

CXEMA 1.

ПАТОГЕНЕЗ ЭНДОМЕТРИОЗА
Статистические данные этой проблемы чрезвычайно разнятся [1-3, 7, 17, 29], хотя во всех научных публикациях и в практической деятельности отмечается прогрессивный рост случаев эндометриоза. Так, его частота в популяции составляет от 7 до 50\%, хирургическая констатация процесса имеет место в 9-11\% случаев. При УзИ диагноз аденомиоза устанавливают в 2,9-5\% случаев, в то время как после гистерэктомии гистологически аденомиоз выявляется в 11-24\% случаев. Среди пациенток, обследовавшихся по поводу нарушений менструального цикла (МЦ), тазовой боли, бесплодия, эндометриоз выявляется у практического большинства - у 23-59\% женщин $[1,2,16,29,30]$.

\section{ПАТОГЕНЕЗ}

Профрессор А.И. Давыдов весьма точно и образно охарактеризовал эндометриоз как «болезнь теорий»: «Чем больше мы знаем об эндометриозе, тем мы меньше знаем об эндометриозе».

Согласно теории R.W. Shau, патогенетическое обоснование развития эндометриоидных гетеротопий включает несколько аспектов (схема 1):

3 наследственные фракторы, связанные с процессами метаплазии и реализующиеся на фоне иммунологических и гормональных нарушений/перестройки;

транспортные фракторы - менструальный рефлюкс, который также может быть связан с гормональными нарушениями.

В дальнейшем развитие гетеротопий может идти по общему сценарию - имплантация, прогрессирующая инвазия, гематогенная и/или лимфогенная диссеминация [7].

Рассматривая патогенез теории метаплазии, следует отметить тот фракт, что она дает понимание того, каким образом могут возникать все известные кисты и кистомы яичника, а также почему в яичниках могут развиваться малые эндометриоидные образования - «глазки» (рис. 1) или крупные опухоли - эндометриомы яичников (рис. 2).

\section{Теория метаплазии}

Всю поверхность яичника покрывает целомический эпителий, который имеет зародышевое происхождение, а именно - парамезонефральное происхождение из мюллерова протока. Из парамезонефрального протока развивается трубный, эндоцервикальный и эндометриальный эпителии. Метаплазированный целомический эпителий является тканевым зачатком всех известных опухолей и опухолевидных образований яичников - серозных, муцинозных и эндометриальных.

Париетальная и висцеральная брюшина тоже имеет мезотелиальное происхождение, но это уже является зрелым мезотелием, хотя и в нем могут присутствовать вкрапления целомического (незрелого) эпителия. Установлены существенные изменения местных 


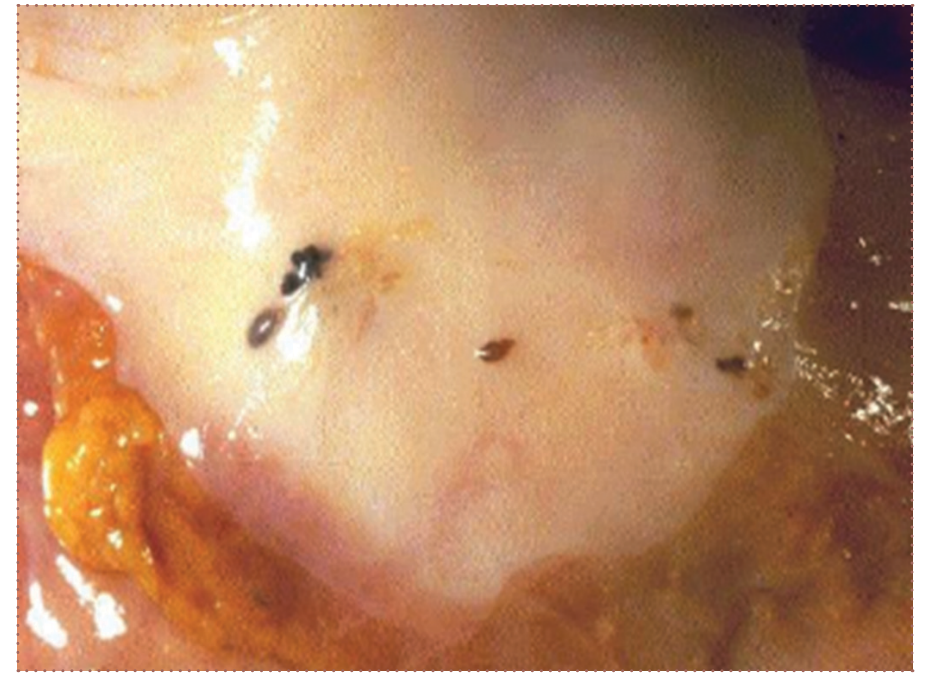

МАЛЫЕ ЭНДОМЕТРИОИДНЫЕ ОБРАЗОВАНИЯ - «ГЛАЗКИ

факторов иммунитета и циркулирующих иммунологических комплексов в крови больных с эндометриозом [22, $23,32]$. Вероятнее всего, эндометриоз является следствием недостаточной функции макрофрагов или сниженной активности естественных клеток-киллеров. Имеются доказательные данные о том [23], что в очагах эндометриоза и в гиперплазированном эндометрии нарушен баланс молекулярно-генетических показателей процессов пролиферации и апоптоза (абсолютно низкий апоптоз и высокая пролиферативная активность).

Имеет место также теория развития эндометриоидной ткани из аномально расположенных эмбриональных зачатков - мюллерова протока [23].

\section{ДИАГНОСТИКА}

Диагностика эндометриоза основывается на следующих критериях:

5 констатация косвенных и клинических признаков;

регистрационные критерии УЗИ (рис. 3), компьютерной или магнитно-резонансной томографии (МРТ), эндоскопии (визуализация) (рис. 1, 2);

морфологическое изучение операционного материала обязательное наличие эпителиального и стромального компонентов, обычно имеющих эндометриальный характер.

\section{КЛИНИЧЕСКИЕ ПРОЯВЛЕНИЯ}

Переходя к характеристике клинических фрорм эндометриоза, необходимо остановиться на дефинициях этой патологии [1-3, 23, 32]. Перечисленные ниже фрормы отражают как клинические характеристики процесса, так и возможность реализации репродуктивной функции.

Различают следующие формы эндометриоза:

5 начальные;

малые;

классический клинический эндометриоз - неблагоприятный эндометриоз (ваd endometriosis);

(5ессимптомное течение - благоприятный эндометриоз (good endometriosis).

Начальные формы эндометриоза - это фрактически его начальные стадии. Имплантаты эндометрия (даже если они и множественные) еще не обладают свойствами инфильтративного роста. При этой фрорме эндометриоза практически у большинства пациенток отсутствует болевой синдром.

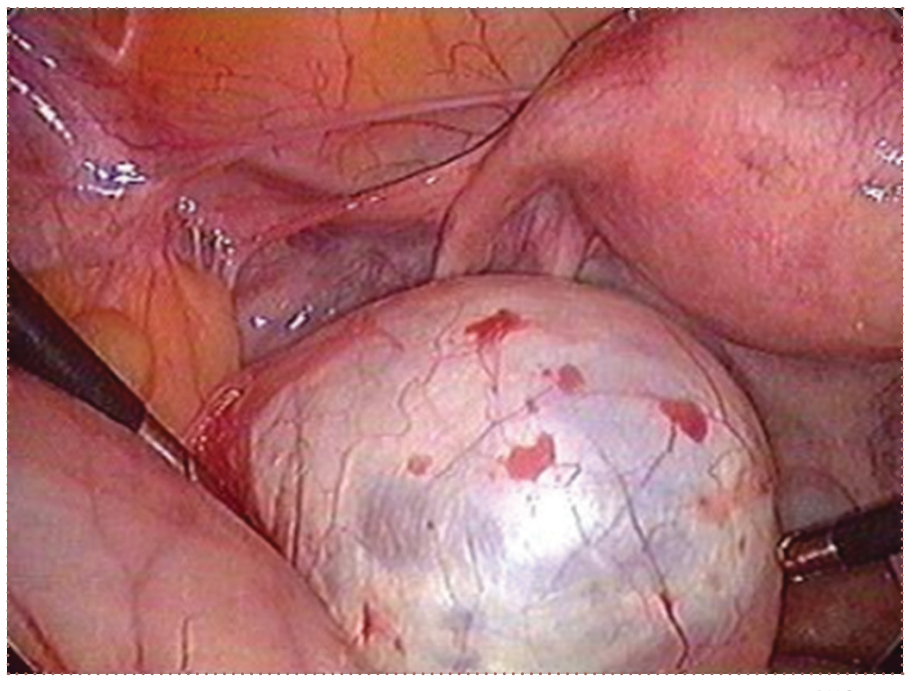

КРУПНЫЕ ОПУХОЛИ - ЭНДОМЕТРИОМЫ ЯИЧнИКОВ

Малые формы эндометриоза. Данная форма указывает лишь на размер эндометриоидных гетеротопий (не более 5 мм в диаметре) и может включать начальные фрормы, а также иметь инфильтративный рост. Наличие болевого синдрома зависит от локализации гетеротопий.

Классический клинический эндометриоз, или неблагоприятный эндометриоз (вad endometriosis) имеет выраженные клинические проявления - нарушения менструальной функции (усиление и удлинение менструальных кровотечений), наличие тазовой боли различной степени тяжести, диспареунии. Первичное бесплодие у таких пациенток диагностируется в 78\% случаев [23]. Ультразвуковая допплерометрия фриксирует изменения в периферической гемодинамике: резистентность стенки маточной артерии и постоянное давление в фазу систолы. Лапароскопия позволяет визуально определить спаечный процесс - эндометриоидные «глазки» с инфильтрацией, эндометриоидные кисты. Имеются данные о нарушении фризиологической регуляции транспортной фрункции труб за счет изменения уровня простагландина $\mathrm{F}_{2 \alpha}$ [10]. Уровень последнего у женщин с эндометриозом повышен в 5 раз из-за стимулирующего влияния на циклооксигеназу 2-го типа в клетках стромы эндометрия.

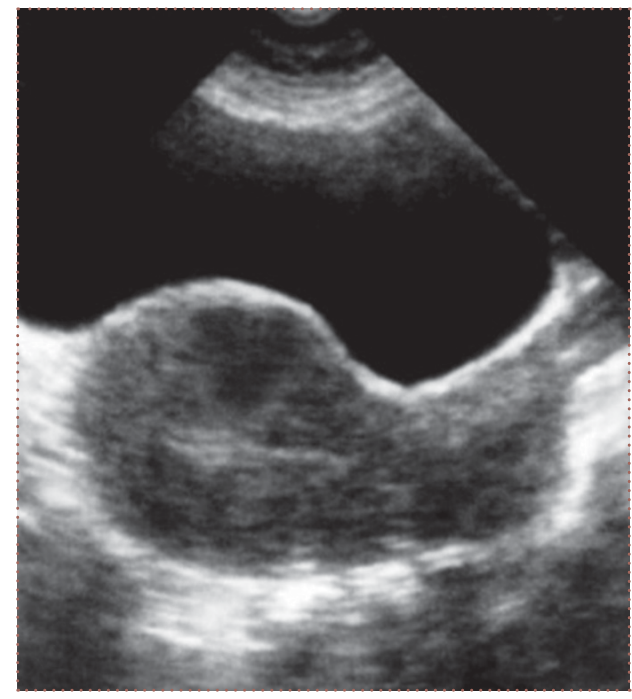

РИС. 3. ЭХОГРАММА МАТКИ С АДЕНОМИОЗОМ 


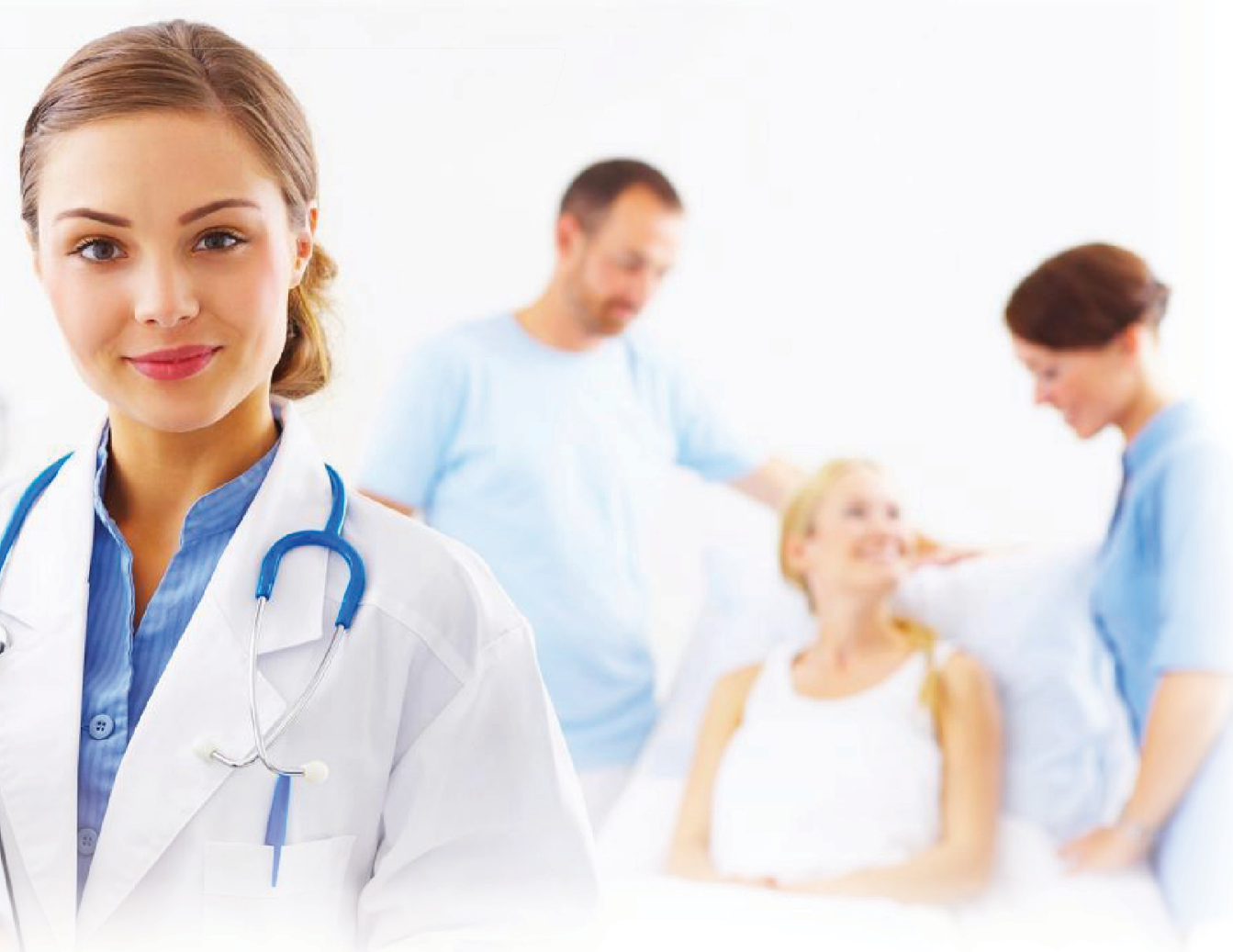

Кроме того, очаги эндометриоза продуцируют факторы роста, цитокины, онкогены при отсутствии экспрессии гена-супрессора р53 (его активация связана с определенными уровнями эндогенного прогестерона), инициируя последующие нарушения и усугубляя имеющийся иммунодесицит [22].

Еще одной особенностью классического клинического эндометриоза является низкий уровень рецепторов прогестерона, что делает бесперспективным лечение прогестинами [23].

Бессимптомное течение, или благоприятный эндометриоз (good endometriosis) характеризуется:

отсутствием клинических симптомов при первичном обращении; диагноз устанавливают в результате проведения ряда исследований;

与 ультразвуковая допплерометрия не выявляет изменений в перифрерической гемодинамике;

фозизологическая регуляция транспортной фрункции труб не нарушена (определяется соотношением простагландинов $\mathrm{E} / \mathrm{F}_{2 \alpha}$ );

пациентки имеют возможность спонтанно забеременеть - первичное бесплодие отсутствует, вторичное составляет до $2 \%$ случаев.

Качественная характеристика этой фрормы эндометриоза заключается в том, что уровень представленности прогестероновых рецепторов многократно (в 9 раз) выше по сравнению с классической клинической его фрормой, чем и объясняется благоприятный эффрект при его лечении синтетическими прогестинами.

\section{ЭНДОМЕТРИОЗ И БЕСПЛОДИЕ}

Существует абсолютно четкая зависимость между возможностью реализации репродуктивных планов и развитием эндометриоза, особенно некоторых его фрорм. Необходимо отметить, что эпителий маточних труб не является местом эндометриоидных имплантов, т.е. сама проходимость маточной трубы сохранна. В основе трубного фактора бесплодия при эндометриозе лежат следующие механизмы:
3 нарушение анатомии маточных труб - наличие спаечного процесса, вызывающего деструкцию труб по их ходу и непроходимость ампулярной части;

при эндометриозе повышается концентрация простагландина $\mathrm{F}_{2 \alpha}$ в эндометриоидной ткани и в сыворотке крови, что обусловливает:

- нарушение транспортной функции маточных труб (физиологическая регуляция транспортной функции определяется соотношением простагландинов $\left.\mathrm{E} / \mathrm{F}_{2 \alpha}\right)$;

- преждевременное угасание желтого тела - недостаточность лютеиновой фразы.

Еще одна особенность, касающаяся проблемы эндометриоза и бесплодия, заключается в том, что развитие эндометриоидных имплантов приводит к преждевременному истощению овариального резерва и развитию синдрома преждевременного истощения яичников [4].

Согласно научным публикациям, эндометриоз ассоциируется с риском развития рака яичников (относительный риск [OP] 1,32) - при применении комбинированных оральных контрацептивов (KOK) на протяжении 10 мес отмечается статистически доказанное снижение риска (ОР 0,21).

\section{ЛЕЧЕНИЕ}

Лечение эндометриоза - весьма длительный процесс, позитивные результаты которого не всегда достижимы. И тем не менее, намечая план лечения, врачи должны понимать, что в настоящее время существуют два возможных пути терапии медикаментозный и хирургический.

Цели лечения эндометриоза:

1. Уменьшение болевого синдрома.

2. Локализация/коррекция спаечного процесса.

3. Препятствование распространению очагов эндометриоза.

4. Нормализация МЦ, ликвидация диспареунии.

5. Вступление в беременность.

Рассматривая вопрос методов лечения, необходимо отметить возможность комбинации их различных вариантов $[1,2,11,16-18]$ : 
медикаментозное лечение - агонисты гонадотропных рилизинг-гормонов (аГнРГ) + хирургические методы (лапароскопия, мини-лапаротомия, крестцово-маточная денервация и пресакральная нейрэктомия при выраженном болевом синдроме);

хирургическое лечение + медикаментозные методы (аГнРГ, прогестины);

э медикаментозное лечение - прогестины, низкодозированные КОК с селективными прогестинами.

Анализируя возможные варианты лечения, необходимо отметить, что все они зависят от клинической ситуации, профрессионализма врача, оснащенности и возможностей медицинского учреждения, а также материального положения пациентки.

Остановимся на реальных возможностях всех применяемых методов лечения, ожидаемых результатах и/или их осложнениях и побочных эффректах.

Лапароскопия

Этот метод хирургического вмешательства имеет четкие показания при наличии:

эндометриом;

аденомиоза, сопровождающегося обильными кровотечениями и анемизацией;

выраженного болевого синдрома;

в случае непереносимости или неэфрфективности гормональных препаратов.

По данным литературы [2, 4, 16, 17], из всех вариантов современных лапароскопических технологий только безгазовая и однопортовая лапароскопия снижает риск спайкообразования. Таким образом, надежды на хирургическое лечение спаечного процесса при обширном наружном эндометриозе и уменьшение спайкообразования в послеоперационном периоде можно отнести к желаемым, а не реальным результатам.

Агонисты гонадотропных рилизинг-гормонов

Концепция медикаментозного лечения с применением аГнРГ основана на следующих позитивных моментах этой терапии:

влияние на репродуктивную ось гипоталамус - гипофиз - яичники: подавление продукции ГнРГ гипоталамуса $\rightarrow$ снижение синтеза гонадотропных гормонов гипофиза $\rightarrow$ «молчание» яичников;

о отсутствие циклической работы яичников $\rightarrow$ уменьшение пролиферативных, инфрильтративных и сосудистых составляющих эндометриальных гетеротопий;

м минимизация побочных эффектов (влияние на метаболизм через сродство к различным рецепторам).

Прекрасная возможность применения разнообразных препаратов аГнРГ в предоперационной подготовке и послеоперационном периоде давно апробирована. Вместе с тем нецелесообразно использование аГнРГ более 6 мес в

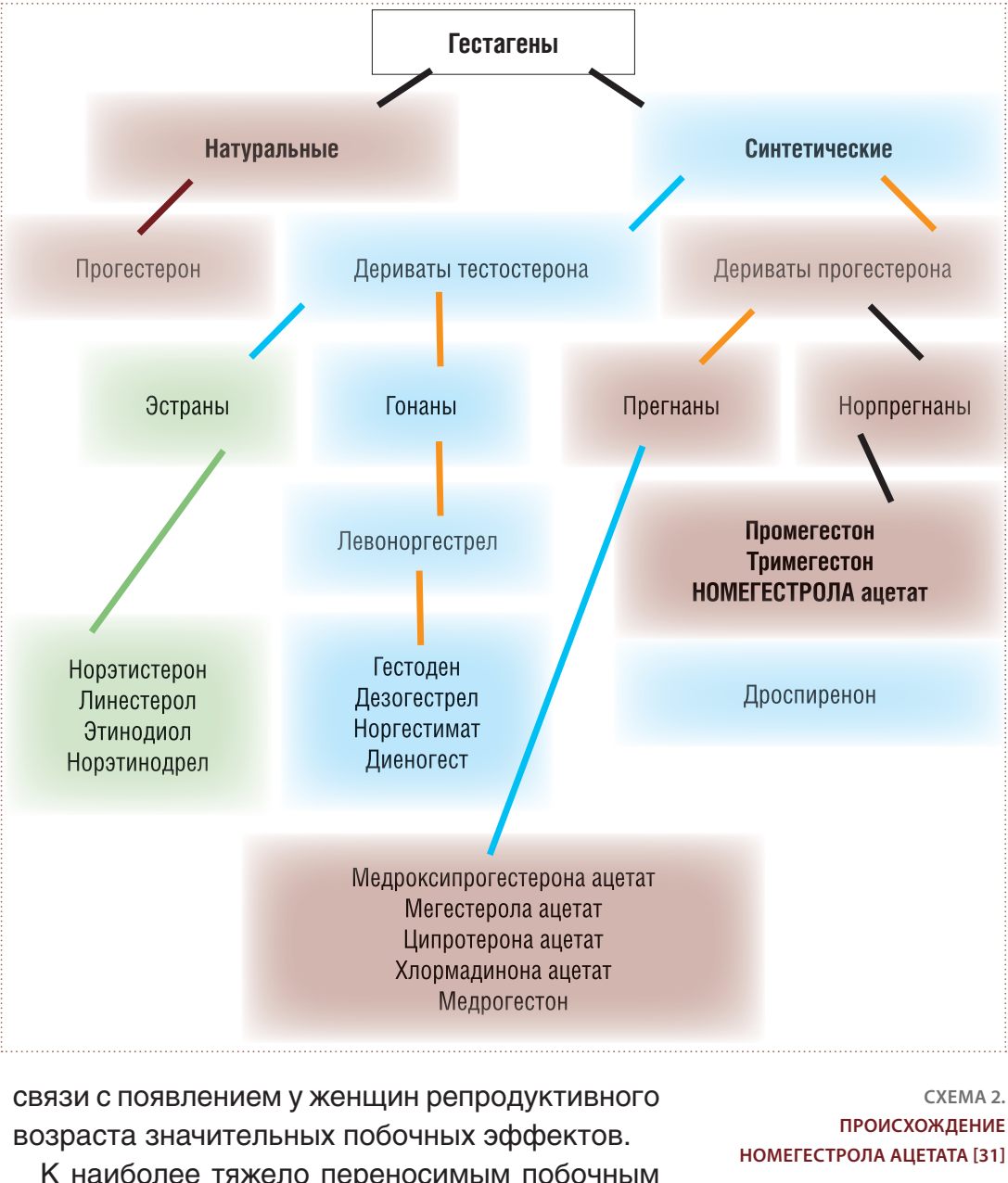

K наиболее тяжело переносимым побочным эффректам аГнРГ с возможным отказом от их применения относят (Perino A. et al., 1993):

с сухость во влагалище;

чу чуство жжения во влагалище;

з диспареунию;

смену настроения, депрессию.

Длительное применение аГнРГ (> 6 мес) может привести также к ингибированию синтеза гонадотропинов и формированию резистентных яичников, потере костной ткани за счет губчатого вещества и развитию других симптомов эстрогендефицита [9, 15, 33].

Комбинированные оральные контрацептивы

Современные однофразные КОК можно применять в разных режимах - циклическом и пролонгированном $(24+4,42+7,126+7)$. Основное достоинство современных КОК - наличие в их составе селективных прогестинов, обладающих выраженными антипролиферативными свойствами. Однако высокая суммарная доза эстрогенов при пролонгированных приемах, связанные с этим особенности переносимости препарата и наличие маточных геморрагий обусловливают необходимость рекомендовать КОК на последующих этапах ведения пациенток с эндометриозом, после выполнения основного лечения.

\section{Прогестины}

Все нижеперечисленные прогестины (норэтистерона ацетат, медроксипрогестерона ацетат, дидрогестерон, 17-окспипрогестерона 


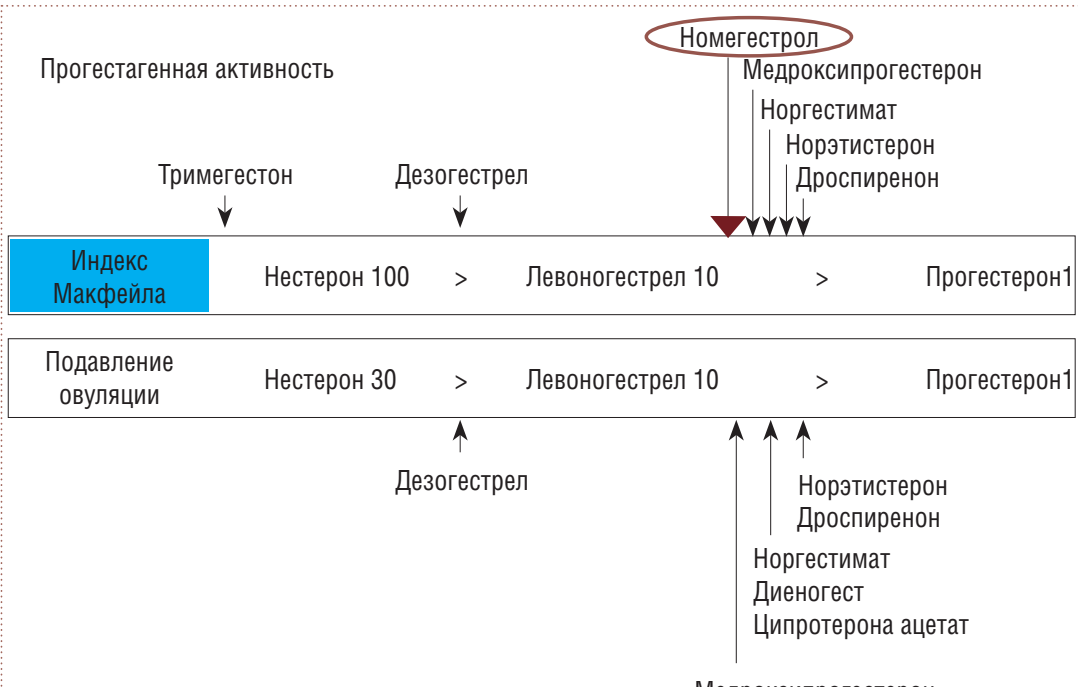

CXEMA 3.

СРАВНЕНИЕ ПРОГЕСТАГЕННОЙ АКТИВНОСТИ ГЕСТАГЕНОВ (SITRUK-WARE R., 2004)

капромат, микронизированный прогестерон, левоноргестрелвысвобождающая система) хотя и применяются в гинекологической практике, но не имеют каких-либо определенных оптимальных свойств [9, 19, 24, 27, 31, 34].

При лечении эндометриоза прогестины должны обладать оптимальными/идеальными свойствами:

5 антигонадотропным эффректом;

(5 антипролиферативным действием;

активировать апоптоз (цитохром $\mathrm{P}_{450 \mathrm{~A}} \mathrm{~B}$ очагах эндометриоза);

возможностью долговременного применения с минимальными метаболическими эффектами и в минимальной дозе, а также возможностью реализации репродуктивных планов.

ТАБЛИЦА 1

\begin{tabular}{|c|c|c|c|c|c|}
\hline \multirow[b]{2}{*}{ Прогестин } & \multicolumn{5}{|c|}{ Рецепторы стероидных гормонов } \\
\hline & Прогестерона & Андрогенов & Эстрогенов & $\begin{array}{c}\text { Глюкокор- } \\
\text { тикоидов }\end{array}$ & $\begin{array}{c}\text { Минерало- } \\
\text { кортикоидов }\end{array}$ \\
\hline $\begin{array}{l}\text { Натуральный } \\
\text { прогестерон }\end{array}$ & 50 & 0 & 0 & 10 & 100 \\
\hline Дидрогестерон & 75 & 0 & & & \\
\hline $\begin{array}{l}\text { Ципротерона } \\
\text { ацетат }\end{array}$ & 90 & 6 & 0 & 6 & 8 \\
\hline $\begin{array}{l}\text { Медрокси- } \\
\text { прогестерона } \\
\text { ацетат }\end{array}$ & 115 & 6 & 0 & 29 & 160 \\
\hline Промегестрон & 100 & 0 & 0 & 3 & 53 \\
\hline $\begin{array}{l}\text { Номегестрол } \\
\text { (Лютенил) }\end{array}$ & 125 & 6 & 0 & 6 & 0 \\
\hline & $\begin{array}{l}\text { одство NOM } \\
\text { ем у прогесте }\end{array}$ & рецептора & $\begin{array}{l}\text { естерона } \\
\text { ше, чем у д }\end{array}$ & $\begin{array}{l}\text { раза выше, } \\
\text { гестерона }\end{array}$ & \\
\hline
\end{tabular}

ТАБЛИЦА 1.

ОТНОСИТЕЛЬНОЕ СРОДСТВО РАЗЛИЧНЫХ ПРОГЕСТИНОВ К РЕЦЕПТОРАМ СТЕРОИДНЫХ ГОРМОНОВ (\%) (KUHL H., 2000)
Прогестины (синтетические гестагены) имеют различное происхождение, мы же остановимся на группе дериватов прогестерона - норпрегнанах (схема 2). Норпрегнаны - производные $17 \alpha$-гидроксипрогестерона - получили свое название в результате удаления радикала $\mathrm{CH}_{3}$ в позиции C-19 (nor - no radical), а добавление двойной связи между С-6 и С-7 в молекуле гидроксипрогестерона повысили его прогестагенную активность [26]. В регистре препаратов Украины зарегистрирован представитель этой группы - номегестрола ацетат (NOMegestrol ACetat, NOMAC), являющийся активным веществом препарата Лютенил. Номегестрола ацетат успешно использовали для лечения некоторых видов гинекологической патологии (менструальные нарушения, дисменорея, предменструальный синдром), а также в качестве компонента заместительной гормональной терапии (ЗГТ) в комбинации с эстрадиолом для уменьшения выраженности менопаузальных симптомов [8, 12, 15, 20, 21, 25]. Данный препарат был одобрен в Европе в качестве монотерапии для лечения лиц с менструальными расстройствами, заболеваниями матки и меноррагией, а в комбинации с эстрогенами - для лечения пациенток с менопаузальными симптомами [28, 35, 36]. Создан и применяется также контрацептив - комбинация натурального эстрогена и номегестрола ацетата [35].

Сотрудники кафедры акушерства и гинекологии № 1 НМАПО им. П.Л. Шупика, начиная с 2010 г., проводили клиническое внедрение данного препарата в гинекологическую практику.

Прогестагенная активность Лютенила прошла исследования согласно протоколу по индексу Максрейла (схема 3) - доза прогестина, вызывающая секреторную трансформацию эндометрия и обладающая антигонадотропной активностью [24, 25].

Установлено влияние Лютенила на шеечную слизь: под его действием происходит потеря вязкости, отсутствует феномен папоротника, повышается уровень гликопротеидов.

Действие Лютенила на эндометрий имеет следующие точки приложения:

с секреторную трансорормацию;

3 антипролиферативное действие - снижение синтеза ДНК и рецепторов эстрадиола, повышениеактивности 17 $\beta$-эстрадиолдегидрогеназы.

Значительно позже $[13,14,18,36]$ появились данные об антигонадотропном действии Лютенила. Имеется четкая дозозависимая ингибиция овуляции при назначении Лютенила с 5-го по 25-й день МЦ:

при применении препарата в дозе 1,25 мг/сут происходит ингибиция овуляции, но отмечается рост фролликула;

(3ютенил в дозе 2,5-5 мг/сут вызывает супрессию овуляции и роста фолликула.

В реализации биологических эфффектов прогестинов большое значение имеет их связь с рецепторами стероидных гормонов (табл. 1) [24].

Отсутствие сродства к андрогенным, минералокортикоидным, эстрогенным и глюкокортикоидным рецепторам (отличие от норэтистерона ацетата, медроксипрогестерона ацетата, линестренола) свидетельствует о метаболической 
нейтральности Лютенила. Отличием Лютенила от прогестерона, дидрогестерона является наличие у него антигонадотропного действия, более выраженное прогестагенное и антиэстрогенное действие (более выраженный антипролиферативный эффект).

Доказательная база безопасности Лютенила $[11,12,15,21,25,27,33,35,36]$

Нейтральный метаболический эффрект «чистых» прогестагенных молекул, к которым относится и номегестрола ацетат (Лютенил), объясняет его лучшую переносимость.

Препарат оказывает влияние на метаболические фракторы риска развития сердечнососудистых заболеваний:

нейтральное или положительное действие на липидный профиль (рис. 4);

нейтральное влияние на уровень глюкозы (рис. 5);

положительное действие на уровни С-реактивного протеина и гомоцистеина;

отсутствие отрицательного влияния на гемостатические факторы.

Анализируя данные литературных источников, касающихся доказательной базы безопасности приема прогестина номегестрола ацетата (Лютенила), можно сделать выводы об отсутствии у него чувствительности к:

глюкокортикоидным рецепторам - не влияет на углеводный обмен и массу тела;

минералокортикоидным рецепторам - не повышается артериальное давление и нет задержки жидкости (отеков);

андрогенным рецепторам - отсутствие возможности проявления акне, гирсутизма, себореи;

э эстрогенным рецепторам - не оказывает отрицательного влияния на молочную железу.

Фармакокинетика Лютенила заключается в том, что у этого препарата отмечается быстрое всасывание в желудочно-кишечном тракте, достигающее пика в плазме крови в течение 2 ч. Длительный период полувыведения (в среднем 40 ч) и связывание в плазме только с альбумином обеспечивает постоянное гормональное насыщение и постоянный уровень номегестрола ацетата в плазме крови в течение суток.

Существующими клиническими исследованиями $[10,11,28,33]$ доказана эффрективность применения 5 мг Лютенила в лечении менструальных расстройств у женщин различных возрастных групп, лейомиомы, эндометриоза, коррекции мастодиний и мастопатий.

Введение препарата Лютенил в гинекологическую практику (на клинической базе кафедры акушерства и гинекологии № 1 НМАПО им. П.Л. Шупика) мы начали с коррекции клинической симптоматики и метроррагий у пациенток с эндометриозом. Критерии включения препарата номегестрола ацетата и ожидаемый лечебный эффрект при эндометриозе основывались на следующих его свойствах:

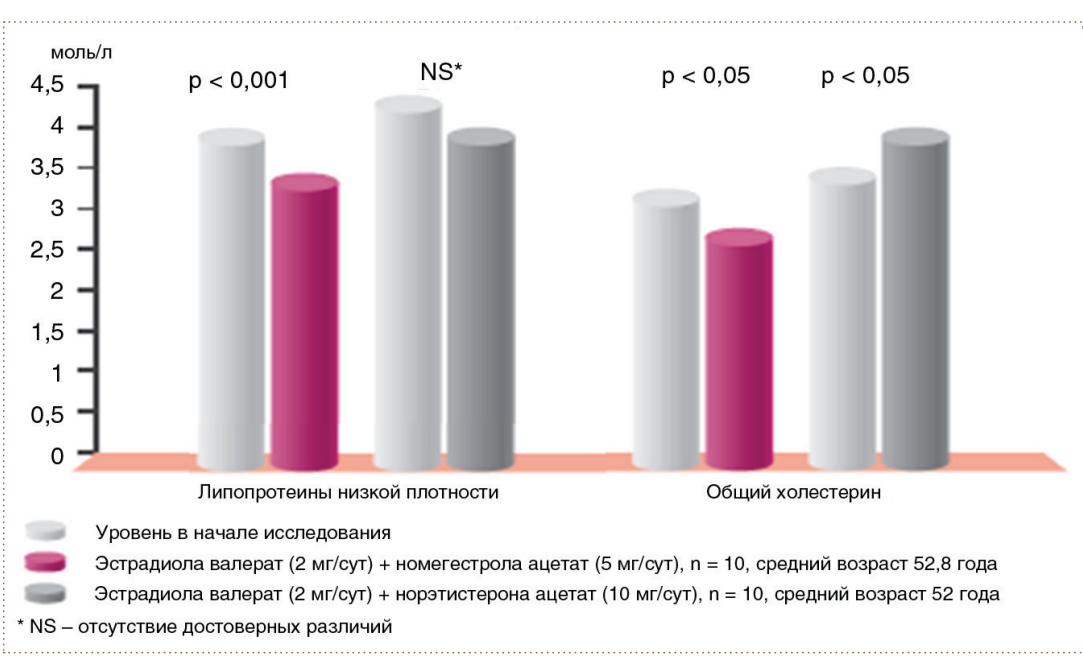
(DORANGEON P., THOMAS J.L., GILLERY P. ET AL., 1992)

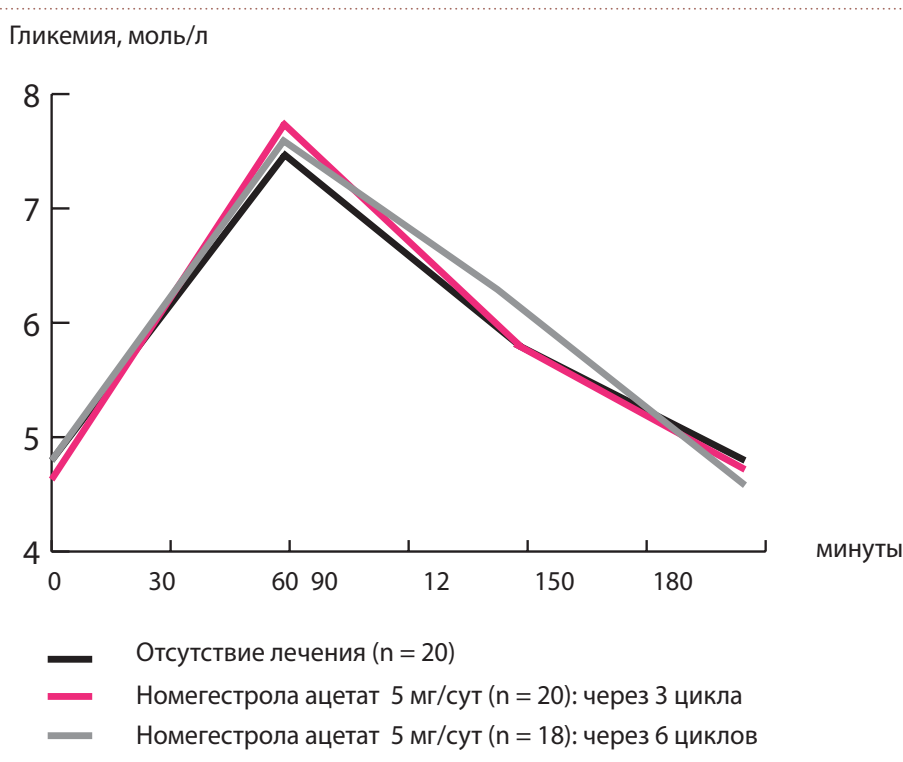
(DORANGEON P., THOMAS J.L., CHOISY H. ET AL., 1993).
До лечения

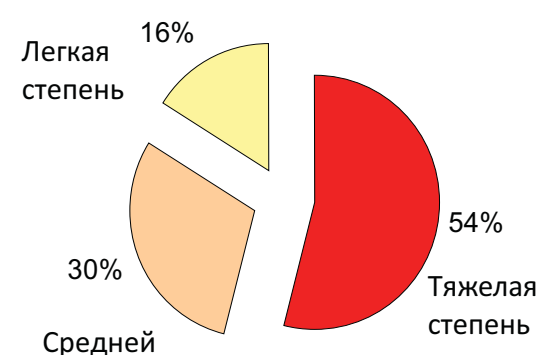

Средней

тяжести

\section{После лечения Лютенилом}

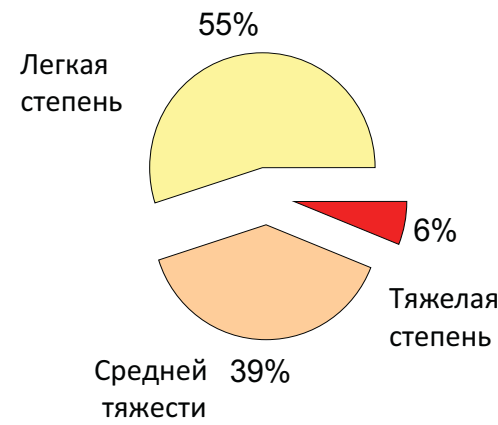

РИС. 6. КЛИНИЧЕСКИХ СИМПТОМОВ ЭНДОМЕТРИОЗА (СОБСТВЕННЫЕ ДАННЫЕ) 
ТАБЛИЦА 2

\section{Протокол МРТ № 1331}

До лечения (20.05.2011)

Правый яичник увеличен в размерах за счет кистоподобного образования округлой формы с четкими контурами, с толстой капсулой, с неоднородным содержимым, гиперинтенсивным и резко гипоинтенсивным сигналом на Т2 33 , T2 FSat - последовательности, слабогиперинтенсивным и изоинтенсивным сигналом на Т1 33, размерами 29×27×23 мм - с большой вероятностью киста со сгустком крови.

Левый яичник с четкими контурами $26 \times 23 \times 24$ мм. В толще яичника визуализируется кистоподобное образование округлой формы с четкими контурами, с толстой капсулой, со слабо гиперинтенсивным МР-сигналом на T2, T1 33, T2 FSat - последовательности, размерами $10 \times 8 \times 8$ мм - с большой вероятностью киста с геморрагическим содержимым.

Заключение: МР-признаки кистоподобного образования с неоднородным содержимым (с большой вероятностью киста желтого тела, нельзя исключить эндометриоз), кистоподобное образование левого яичника (с большой вероятностью киста желтого тела, нельзя исключить эндометриоз), фоолликулярные кисты левого яичника

\section{Протокол МРТ № 2437}

После лечения (13.09.2011)

Правый яичник размером $30 \times 20 \times 22$ мм, в структуре яичника определяется участок гиперинтенсивного МР-сигнала - на Т1-ВИ и неоднородного гипоинтенсивного - на Т2-ВИ, Т2 FSat, неправильной формы $14 \times 10 \times 9$ мм - характерно для геморрагического содержимого. Перифокально субкапсулярно визуализируются тонкостенные округлые кисты 4-6 мм в диаметре с жидкостным содержимым (с гиперинтенсивным MP-сигналом на Т2-BИ, T2 FSat, гипоинтенсивным на Т1-ВИ).

Левый яичник - 32×27×20 мм, субкапсулярно визуализируется тонко- и толстостенные кисты 4-11 мм в диаметре с жидкостным содержимым.

Заключение: МР-признаки функциональных кист яичников (фолликулярных и кист желтого тела), кистозное образование правого яичника с геморрагическим содержимым, возможно кровоизлияние в кисту желтого тела, эндометриома
ТАБЛИЦА 2. ДАННЫЕ МРТ

ПАЦИЕНТКИ С ПЕРВИЧНО ДИАГНОСТИРОВАННЫМ НАРУЖНО-ВНУТРЕННИМ ЭНДОМЕТРИОЗОМ ДО И ПОСЛЕ ЛЕЧЕНИЯ влияние на гипоталамо-гипофизарнояичниковую ось и снижение овуляторного пика лютеинизирующего и фролликулостимулирующего гормонов (дополнительный эфффект препарата позволяет применять его с целью контрацепции);

подавление созревания фролликулов и снижение овариальной секреции эстрогенов;

5 антипролиферативное действие - эндометриальная супрессия (топическая и эктопическая).

В клиническое исследование вошли пациентки в возрасте от 23 до 44 лет с ранее диагностированным наружно-внутренним эндометриозом либо диагностика производилась нами при первичных визитах женщин. Режим назначения Лютенила - с 5-го по 25-й день МЦ в дозе 5 мг/ сут (1 таблетка). Документальную верификацию процесса осуществляли с помощью УЗИ с допплеровской приставкой либо путем МРТ. При наличии образований в яичниках исследовали онкомаркеры СА-125 и НЕ-4. Следует отметить, что диагностическая триада - исследование этих двух онкомаркеров в сочетании с допплер-УЗИ - относится к ранним возможностям диагностики неопластических процессов в яичниках. При активных пролиферативных процессах в очагах эндометриоза обнаруживается существенное повышение уровня СА-125 (неспецифический маркер), и в дальнейшем при эфффективном лечении его уровень снижается. В то же время у пациенток фриксируется крайне низкий уровень высокоспецифичного раннего онкомаркера HE-4.
Также нами оценивались основные клинические признаки эндометриоза (тазовая боль, дисменорея, гиперполименорея, диспареуния) с их детализацией по степени тяжести (легкая, среднетяжелая и тяжелая).

Результаты динамики клинических симптомов эндометриоза на фоне лечения Лютенилом в течение 3 мес таковы:

тазовая боль: 80\% пациенток отмечали тяжелую степень болевых ощущений, легкая степень отсутствовала. Через 3 мес тяжелая степень наблюдалась у 10\% женщин, среднетяжелая - у $30 \%$, легкая - у $60 \%$;

дисменорея: до лечения легкая степень отсутствовала, средняя степень тяжести имелась у $25 \%$ лиц, тяжелая - у $75 \%$. Через 3 мес тяжелую степень зафиксировали у 3\% пациенток, среднетяжелую - у 40\%, легкую - у 60\%.

гиперполименорея: до лечения легкая степень была обнаружена у $2 \%$ участниц исследования, средняя - у 38\%, тяжелая - у $60 \%$. Через 3 мес тяжелая степень отсутствовала, среднетяжелая выявлена в $67 \%$ случаев, легкая - в 33\%.

диспареуния: до лечения легкая степень наблюдалась у $60 \%$ женщин, средней тяжести - у 40\%, тяжелая степень отсутствовала. Через 3 мес среднетяжелая степень имелась у 10\% пациенток, легкая - у 90\%.

Совокупность выраженности всех клинических симптомов эндометриоза и их динамика при лечении Лютенилом представлена на рис. 6.

Ниже приводятся данные МРТ пациентки К., 24 лет, с первично диагностированным наружновнутренним эндометриозом (табл. 2). Отмечается уменьшение образования в правом яичнике в 2 раза: при первичном исследовании выявлено образование размером 29-27-23 мм, при вторичном - 14-10-9 мм.

При длительном лечении чистыми прогестинами могут отмечаться незначительные межменструальные кровотечения, которые при приеме Лютенила исчезают по мере лечения (возможно также добавление одной дополнительной таблетки препарата в течение 2-3 дней). Симптомы вагинальной сухости, выраженных проявлений диспареунии купировали колпотрофином - препаратом проместрина (эстроген, действующий исключительно локально). У всех пациенток на протяжении лечения не выявлено изменений параметров артериального давления и колебания веса.

\section{ВЫвОды}

Эндометриоз - единственный в своем роде дисгормональный процесс, характеризующийся способностью к инфильтративному росту и метастазированию. Выбор методов лечения эндометриоза требует дополнительной и тщательной диагностики. 
Среди медикаментозных методов терапии в приоритете остаются чистые прогестины, которые имеют минимальные метаболические эффректы и обладают высокой фрармакологической активностью. Селективный прогестин номегестрола ацетат (Лютенил) имеет все вышеперечисленные характеристики и быстро купирует клинические проявления эндометриоза. Возможность его долговременного применения в минимальной дозе и с минимальными побочными эффектами создает дополнительные преимущества для пациенток, которые еще не реализовали свои репродуктивные планы.

\section{ЛИТЕРАТУРА}

1. Адамян Л.А., Кулаков В.И. Эндометриозы. - М.: Медицина, 1998. - 316 с.

2. Андреева Е.Н. Распространенные формы генитального эндометриоза. Медико-генетические аспекты, диагностика, клиника, лечение и мониторинг больных.: Автореф. дис. канд. мед. наук. - М. - 1997. - 50 с.

3. Баскаков В.П., Цвелев Ю.В., Кира Е.Ф. Диагностика и лечение эндометриоза на современном этапе. - СПб.: НЛ, 1998. - 452 c.

4. Всемирный конгресс по менопаузе. - Рим, 2011. Тезисы. - С.109.

5. Кулаков В.И., Адамян Л.А., Аскольская С.И. Гистерэктомия и здоровье женщины. - М.: Медицина, 1999. - 312 с.

6. Справочник по акушерству, гинекологии и перинатологии / Под ред. акад. РАМН Г.М. Савельевой. - М.: МИА, 2006. - С. 518-526.

7. Хмельницкий О.К. Патоморфологическая диагностика гинекологических заболеваний. - СПб.: СОТИС, 1994. - 480 с.

8. Bazin B., Thevenot R., Bursaux C. et al. Effect of nomegestrol acetate, a new 19-nor_progesterone derivative, on pituitary ovarian function in women. Br J Obstet Gynaecol 1987 Dec; 94 (12): 1199-1204.

9. Bullock L.P., Bardin C.W. Androgenic, synandrogenic, and antiandrogenic actions of progestins. Ann N Y Acad Sci 1977 Mar 11; 286: 321-330

10. Cohen A., Engelmann P., Erny R. et al. Lutenyl and menstrual disorders: a hospital study. Rev Fr Gynecol Obstet 1988 Nov; 83 (11): 741-743.

11. Cohen A. Multicenter study of the clinical use of nomegestrol acetate in outpatients. Contracept Fertil Sex (Paris) 1994; 21: 417-427.

12. Conard J., Basdevant A., Thomas J.L. et al. Cardiovascular risk factors and combined estrogen_progestin replacement therapy: a placebo_controlled study with nomegestrol acetate and estradiol. Fertil Steril 1995 Nov; 64 (5): 957-962.

13. Couzinet B., Young J., Brailly S. et al. The antigonadotropic activity of progestins (19-nortestosterone and 19-norprogesterone derivatives) is not mediated through the androgen receptor. J Clin Endocrinol Metab 1996 Dec; 81 (12): 4218-4223.

14. Couzinet B., Young J., Kujas M. et al. The antigonadotropic activity of a 19-norprogesterone derivative is exerted both at the hypothalamic and pituitary levels in women. J Clin Endocrinol Metab 1999 Nov; 84 (11): 4191-4196.
15. De Leo V., la Marca A., Morgante G. et al. Comparison of two HRT regimens with bimonthly and monthly progestin administration in postmenopause.Maturitas 1999 Jan 4; 31 (2): 171-177.

16. Donnez J., Nisolle M. Laparoscopic menegment of large ovarian endometrial cysts // J. Gynecol. Surg. - 1991. - Vol. 7. P. 163-167.

17. Donnez J., Nisolle M., Casanas-Roux F. et al. Endometriosis rational for surgeri. The current status of endometriosis. - 1993. - P. 385-395.

18. Duc I., Botella J., Bonnet P., et al. Antiandrogenic properties of nomegestrol acetate. Arzneimittelforschung 1995 Jan; 45 (1): 70-74.

19. Dusterberg B., Humpel M., Speck U. Terminal half_lives in plasma and bioavailability of norethisterone, levonorgestrel, cyproterone acetate and gestodene in rats, beagles and rhesus monkeys. Contraception 1981 Dec; 24 (6): 673-683.

20. Ezan E., Benech H., Bucourt R. et al. Enzyme immunoassay for nomegestrol acetate in human plasma. J Steroid Biochem Mol Biol 1993 0ct; 46 (4): 507-514.

21. Fraser D.I., Padwick M.L., Whitehead M.I. et al. The effects of the addition of nomegestrol acetate to postmenopausal oestrogen therapy. Maturitas1989 Mar; 11 (1): 21-34.

22. Gleicher N., Dmowski W.P., Siegel I. Lymfocyte subsets in endometriosis. Obstet Gynecol 1984; 63: 463.

23. Kennedy S. The genetics of endometriosis//J Reprod Med 1998: 43; 263-268.

24. Kuhl H. Comparative pharmacology of newer progestogens. Drugs 1996 Feb; 51 (2): 188-15.

25. Kumar N., Koide S.S., Tsong Y. et al. Nestorone: a progestin with a unique pharmacological profile. Steroids 2000 Oct_Nov; 65 (10-11): 629-636.

26. Paris J., Thevenot R., Bonnet P. et al. The pharmacological profile of TX 066 (17 alpha-acetoxy-6-methyl19-nor-4,6-pregnadiene-3,20-dione), a new oral progestative Arzneimittelforschung 1983; 33 (5): 710-715.

27. Pasqualini J.R. Progestins and breast cancer. Gynecol Endocrinol 2007 0ct; 23 Suppl. 1: 32-41.

28. Persiani P., Radici E., Lombardi P.A. et al. Use of nomegestrol acetate in the treatment of menstruation disorders: our experience in 56 cases. Minerva Ginecol 1997 Apr; 49 (4): 181-185.

29. Redwin D.B. Laparoscopic approach to the diagnosis of endometriosis. Матер. межд. конгресса. - М. - 1996. - С. 128-136.

30. Sampson J.A. Perforating hemorrhagic (chocolate) cysts of the ovary. Arch. Surg. - 1991. - P. 245-323.

31. Schindler A.E., Campagnoli C., Druckmann R. et al. Classification and pharmacology of progestins. Maturitas 2003 Dec 10; 46 Suppl. 1: S7-16.

32. Shaw R.W. Atlas of endometriosis. The Parthenon Publishing Group. - 1993. - P. 31-32.

33. Sitruk-Ware R. New progestogens: a review of their effects in perimenopausal and postmenopausal women. Drugs Aging 2004; 21 (13): 865-883.

34. Sitruk-Ware R. Pharmacological profile of progestins. Maturitas 2004 Apr 15; 47 (4): 277-283.

35. Sitruk-Ware R. New progestagens for contraceptive use. Hum Reprod Update 2006 Mar_Apr; 12 (2): 169-178.

36. Stefano Lello Drugs 2010: 70 (5): 541-559. 